\title{
RARSEENDER
}

Trascender, Contabilidad y Gestión Núm. 9 (septiembre - diciembre del 2018). ISSN: 2448-6388. Universidad de Sonora. Departamento de Contabilidad. Reserva de Derechos 04-2015-04172070800-203.

\section{Profesionalización de las Entidades de Fiscalización Superior}

Professionalization of Supreme Audit Institutions

$$
\text { Ramón Rendón Padilla }{ }^{1}
$$

\section{Resumen}

En la actualidad las Entidades de Fiscalización Superior (EFS) locales en México, se encuentran en la necesidad de vivir renovaciones constantes en sus estructuras, diseño institucional y desarrollo de capacidades para ser efectivas en su cobertura de fiscalización a la administración de los Estados.

En el ámbito internacional y nacional existen organizaciones que las delinean como EFS, emitiendo normatividad para su desempeño y lo más importante, ser agentes de cambio en el ejercicio de recursos públicos, traduciendo sus resultados en beneficios para la sociedad en general.

La profesionalización de las EFS, debe ser una forma de vida, sino es que una obligación, adoptando las mejores prácticas, que debe relacionarse con el apego a la legalidad, eficacia, eficiencia y economía en el uso de los recursos públicos, mejorando así el desempeño gubernamental y la rendición de cuentas; también incluye la concepción y el contexto de la profesionalización como eje clave para generar el desarrollo de capacidades en las EFS para lograr el dominio de un saber legitimado, capacidad para diagnosticar problemas propios y encontrar por sí mismo soluciones; autonomía y responsabilidad individual con respecto a su tarea, y responsabilidad colectiva sobre el rendimiento de las EFS.

Palabras clave: Entidades de Fiscalización Superior, profesionalización.

DOI: https://doi.org/10.36791/tcg.v0i9.37

Recibido: 15 de junio de 2018.

Aceptado: 24 de agosto de 2018.

\footnotetext{
${ }^{1}$ Ramón Rendón Padilla. Contador Público. Auditor Supervisor en el Instituto Superior de Auditoria y Fiscalización (ISAF) en el Estado de Sonora. Correo: ramonren1@hotmail.com
} 


\section{Abstract}

Nowadays, the local Supreme Audit Institutions (SAI) in Mexico need constant renovations in their structures, institutional design and capacity development to be effective in their audit coverage of the State administration.

Internationally and nationally, there are organizations that establish regulations for the SAI, issuing normativity for their performance and, most importantly, being agents of change in the exercise of public resources, translating their results into benefits for society in general.

The professionalization of the SAI must be a way of life, an obligation, adopting the best practices, which should be related to the adherence to legality, effectiveness, efficiency and economy in the use of public resources, improving the government performance and transparency; it also includes the conception and context of professionalization as a key axis to develop capacity in SAI to achieve a legitimate knowledge, ability to diagnose their own problems and find solutions for themselves; autonomy and individual responsibility with respect to their task, and collective responsibility over the performance of SAI.

Keywords: Supreme Audit Institutions, professionalization. 


\section{Introducción}

La fiscalización superior muestra una tendencia hacia un desarrollo constante de profesionalización, ya que la globalización ha logrado una influencia directa en las Entidades de Fiscalización Superior (EFS) nacional y local; por eso, cada entidad se ha ocupado de las prioridades que tiene la capacitación en el capital humano, como uno de los factores clave para alcanzar una verdadera profesionalización.

La tendencia referida resulta pertinente, ya que en los últimos años se han expuesto declaraciones de tipo legal, política y social de gobiernos estatales en México, que han incurrido en desviaciones en el ejercicio de los recursos públicos. Por lo tanto, se considera que si las EFS fortalecen su desarrollo profesional e institucional realizarán una tarea de fiscalización más efectiva, más aún si se toma en cuenta que las EFS son consideradas con éxito cuando generan confianza en el ejercicio de los recursos públicos, bienestar social y económico a la población.

Por otro lado, considerando los aportes de los estados del conocimiento y de la práctica, resulta relevante considerar como una obligación la capacitación técnica y especializada del capital humano, de acuerdo con normatividad nacional e internacional de las EFS, ya que ello da una dirección de confianza y certeza en el desempeño, generando influencia en el ejercicio de recursos públicos.

En los últimos tiempos las EFS en México, han sido cuestionadas respecto a los resultados generados por gobiernos locales estatales, punto decisivo para considerar la profesionalización como delimitante en la aplicación de recursos públicos.

Así pues, el desarrollo de este trabajo se centra en el desarrollo del marco teórico construido hasta el momento y en unas breves conclusiones que plantean un horizonte de estudio y de propuesta para la mejora de profesionalización de las Entidades de Fiscalización Superior (EFS).

\section{Origen y fundamento jurídico de la Entidades de Fiscalización Superior en México}

Los antecedentes de la fiscalización en México pueden ser analizada desde tres etapas históricas: época prehispánica, con el control del tributo por parte del Tlatoani; época colonial con los Tribunales de Cuentas creados en 1453 por las Cortes españolas, vigentes por más de tres siglos; y la del México independiente, donde se suprime el Tribunal Mayor de Cuentas con el establecimiento de la Contaduría Mayor de Hacienda. (Tiscareño Agoitia, 2016) .

En 1824, con la expedición de la Constitución Política de los Estados Unidos Mexicanos, en su Artículo 50, donde se facultó al Congreso general para fijar los gastos generales de la nación, establecer las contribuciones necesarias para cubrirlos, arreglar su recaudación, determinar su inversión y tomar anualmente cuentas al gobierno, se dio lugar a la creación del órgano denominado "Contaduría Mayor de Hacienda” (CMH), a cargo de la Cámara de Diputados. Por primera vez se atribuía la función de fiscalizar al poder legislativo. Esta versión fue sufriendo cambios con el correr de la historia. Se le daban atribuciones y se suprimían otras. Se registraron transformaciones que atendían las necesidades de cada época, hasta llegar a la promulgación de la Constitución política de los Estados Unidos Mexicanos de 1917.

En 1917, con la promulgación de la nueva Constitución se estableció, entre otras cosas, en el Artículo 65 que el Congreso se reuniría el $1^{\circ}$ de septiembre de cada año para celebrar sesiones ordinarias, en las cuales se revisaría la Cuenta Pública del año anterior, que sería presentada a la Cámara de 
Diputados dentro de los diez primeros días de la apertura de sesiones. La revisión no se limitaría a investigar si las cantidades gastadas estaban o no de acuerdo con las partidas respectivas del Presupuesto, sino que se extendería al examen de la exactitud y justificación de los gastos hechos y a las responsabilidades correspondientes.

El 30 de julio de 1999 se publicaron en el Diario Oficial de la Federación reformas a la Constitución para fortalecer las funciones de fiscalización de la Cámara de Diputados en lo que refiere a la cuenta pública. En este paquete de reformas el Articulo 73 daba facultad al Congreso de la Unión para expedir la ley que regulara la organización de la entidad de fiscalización superior de la Federación y las demás que normaran la gestión, el control y la evaluación de los Poderes de la Unión y de los entes públicos federales.

El 29 de diciembre del año 2000, para dar cumplimiento a las reformas constitucionales, se publica la nueva Ley de Fiscalización Superior de la Federación (LFSF), con el objetivo de regular la revisión de la Cuenta Pública y su fiscalización superior, así como otras materias, por mencionar la determinación de las indemnizaciones, fincamiento de responsabilidades por daños y perjuicios a la Hacienda Pública Federal y a otros organismos públicos, las bases y términos de organización y funcionamiento de la entidad encargada de la fiscalización superior, dando lugar al nacimiento de la Auditoría Superior de la Federación (ASF), la entidad superior de fiscalización, así como sus atribuciones específicas como normas, objetivos, funciones, regulaciones, en sí toda la estructura para su funcionamiento. De igual forma esta entidad ha tenido transformaciones en los siguientes años, destacando las atribuciones de independencia técnica y de gestión, pero no de autonomía, ya que actualmente sigue siendo el brazo fiscalizador del Congreso de la Unión.
A la par de la función fiscalizadora a nivel nacional, también se han ido desarrollando las Entidades de Fiscalización Superior Locales en cada entidad federativa, mismas que se formaron por lineamientos aplicables en el ámbito federal, adaptados al ámbito local.

\section{Los entes de fiscalización superior}

En el contexto mundial la Organización Internacional de las Entidades Fiscalizadoras Superiores (INTOSAI, por sus siglas en inglés) ha recomendado la independencia y profesionalización de la auditoria gubernamental externa como componentes clave del éxito, según declaración de Lima, Perú. (Organización internacional de las Entidades Fiscalizadoras Superiores (INTOSAI), 1977)

Desde la segunda década del siglo XXI, las entidades fiscalizadoras superiores están atrayendo una atención significativa por parte de una sociedad que ve en ellas instrumentos esenciales para acreditar que las administraciones públicas son merecedoras de confianza. Sin embargo, esa ciudadanía ya no solo espera de las EFS la certificación de que las cuentas de las administraciones públicas recogen la imagen fiel de su situación económico-financiera y que el gasto se hace de acuerdo con la legalidad vigente, sino que además esperan que respondan a nuevas preguntas y que lo hagan en unos plazos razonables para que las respuestas sean útiles, con el fin de que haya cuestionamientos básicos sobre la esencia del uso de los recursos públicos. (Martínez Argüelles, 2017)

Con base en el fundamento jurídico nacional e internacional y los marcos de referencia consultados, se conceptualiza la Fiscalización Superior como un sistema o conjunto de procesos y procedimientos, que involucran actores, normas e instituciones, cuya función consiste en examinar el uso de los recursos públicos y actos de gobierno. No obstante, también se 
concibe como un instrumento que puede servir para lo siguiente:

- Verificar el apego a la legalidad, eficacia, eficiencia, y economía en el uso de los recursos públicos y actos de gobierno, realizados a través de la administración pública.

- Corregir y mejorar el desempeño gubernamental.

- La rendición de cuentas.

- Modernizar y fortalecer la administración pública para prevenir y disminuir actos de corrupción.

- Detectar, corregir y sancionar prácticas irregulares o ilícitas.

Por esto la mayoría de los países, independientemente de su grado de desarrollo y sus regímenes de gobierno, cuentan con EFS; con esquemas de control externo y vigilancia de la acción de gobierno que constituyen, en la práctica, el principal instrumento de rendición de cuentas y un mecanismo de prevención del fenómeno de la corrupción, en muchos casos. (Ortiz Ramírez \& Cruz Pérez, 2017).

En este sentido, y situados en el propósito anunciado en la introducción del presente trabajo, se asume que las EFS deberían tener la profesionalización como un eje rector para desarrollar una gestión con independencia mental y valores como la integridad, ética y profesionalismo, sin perder de vista la integridad personal de sus miembros y la evolución en sus conocimientos. De ahí la importancia de la profesionalización, ya que como afirman (Montilla Galvis \& Herrera Marchena, 2006) la actitud de independencia mental e imparcialidad respecto a su labor están asociados al grado de profesionalismo.

Este profesionalismo también es comprendido como un factor que guarda relación con el desempeño en la fiscalización, cuyos rasgos principales son la integridad e idoneidad, la rectitud ética y profesional.
De ahí la competencia y pericia que debe poseer el auditor al ser un experto no sólo por sus conocimientos, sino también por la experiencia en el campo del ejercicio profesional de la auditoría.

Además, es preciso señalar que las EFS locales tienen grandes retos, mismos que podrán afrontar con instituciones idóneas para el ejercicio profesional, por mencionar algunos está el informar sobre serios problemas de la rendición de cuentas respecto al destino y calidad de los recursos públicos de los gobiernos; incentivar de forma inteligente a la generación de recursos propios en los estados y municipios; analizar profundamente las deudas de los estados; y por último el reto esencial de las EFS, contar con las capacidades de control y fiscalización del uso de los recursos públicos. (Romero Gudiño, 2016)

De acuerdo con lo anterior, la actividad de fiscalización debería tener dos dimensiones: revelar las deficiencias en la ejecución de los presupuestos públicos (perspectiva del pasado) y recomendar mejoras (perspectiva de futuro). Sin la cooperación del Poder Ejecutivo para corregir las fallas y ejecutar las recomendaciones de mejora, la fiscalización superior no alcanza su eficacia plena, pero también sin EFS profesionalizadas y comprometidas con la misma tarea existe riesgo de no vigilar con pericia el uso más eficiente de los recursos públicos.

\section{Profesionalización}

Al analizar el concepto de profesionalización, la Real Academia Española define como profesionalización al proceso y al resultado de profesionalizar (convertir una actividad o una afición en una profesión); La profesionalización implica una serie de cambios en algo, por lo general con la intención de incrementar su calidad y de alcanzar ciertos estándares.

En continuidad con el punto anterior respecto a la profesionalización de las EFS, es primordial reiterar 
que el auditor, para obligarse al encargo de labores de auditoría, debe tener capacidad y competencia avalada por órganos profesionales de los gremios que los agrupan; tales características lo convierten en una persona en la que se puede depositar plena confianza. Debido a lo anterior, es que se le confieren la capacidad de ser fedatario, facultad concedida por la sociedad para que salvaguarde sus intereses y los de sus organizaciones.

(Figueroa Neri, 2007) señala que esta tarea de fiscalización no podría llevarse a cabo sin un ejercicio profesional de la misma, pues es una tarea que el poder Legislativo deposita en instancias que deberían estar sumamente capacitadas para recuperar y analizar información acerca del uso gubernamental de los recursos públicos. Sin organismos ni profesionales capacitados profundamente para esto, el poder Legislativo no tendría control ni vigilancia del Poder Ejecutivo, ni podría aplicar los procedimientos e instrumentos de control, como comparecencias, votos de censura y sanciones en caso de responsabilidades.

Gabriel Purón Cid, profesor e investigador de la Universidad Estatal de Nueva York, señala:

"El papel de los Entes de Fiscalización Superior (EFS) en América Latina ha contribuido a una mejor gestión de los recursos públicos, lo que, a su vez, está logrando el mejoramiento de la calidad de vida de las personas, mediante el incremento al acceso de bienes y servicios públicos. El trabajo de las EFS en los países de la región ha permitido un progreso técnico, no sólo de la gestión fiscal, sino de los resultados de las funciones de vigilancia en la gestión de las entidades vigiladas, para que su actividad misional sea eficaz y eficiente." (Cid, 2016:71)

Sin embargo, esa gestión más justa y transparente de los recursos, se ha visto impulsada no solo por reformas de índole fiscal, sino también por la capacitación y mayor acceso a la información de los auditores. No obstante, la eficacia en la profesión auditora plantea retos, esto se advierte en los estudios de 2013, sobre los entes de fiscalización superior, realizados por el ( Instituto Mexicano para la Competitividad A.C. (IMCO) y el Centro Universitario de Ciencias Económico Administrativas de la Universidad de Guadalajara (CUCEA-UdG), 2013), cuyos resultados determinaron que las leyes de fiscalización locales tienen fuertes deficiencias en puntos clave del proceso de fiscalización superior.

En la revisión que llevaron a cabo a las leyes de fiscalización superior y a los correspondientes portales de las entidades de fiscalización en el país, encontraron la obligación de establecer Sistemas de servicio civil, en este mismo estudio se da cuenta que solo en 16 de las 32 entidades de fiscalización superior tienen el servicio civil o fiscalizador de carrera.

A raíz de estos hallazgos, en estos estudios referidos se ha enfatizado la necesidad de impulsar el establecimiento de un servicio fiscalizador de carrera, sobre todo porque programas como éste son prioritarios para fomentar la profesionalización del personal de las EFSL, y consecuentemente, reducir los riesgos de interferencia política.

\section{La sociedad del conocimiento y las buenas prácticas de profesionalización}

Con base en el punto anterior, las EFS también deben ser generadoras de conocimiento, aplicar las mejores prácticas y la profesionalización de éstas. Las posibilidades que brindan hoy día las nuevas tecnologías de la información y las comunicaciones, en una sociedad del conocimiento nos facilitan y generan expectativas de mejora en la materia de profesionalización de la tarea fiscalizadora.

La sociedad del conocimiento se caracteriza por el desarrollo y la innovación constante de tecnologías, que han aparejado cambios en los modos de acceso a 
la información. Dentro del ámbito de las organizaciones, ello exigió la puesta en marcha de herramientas de capacitación que dieran respuesta a las exigencias de mayor profesionalización y especialización de los recursos. Al mismo tiempo, en el ámbito estatal, estas transformaciones motivaron a la emergencia de nuevos paradigmas de administración pública en América Latina, que han sido aplicados desde los gobiernos con distinto grado de éxito. (Caruncho \& Aiassa, 2016)

\section{Generación y desarrollo de capacidades}

En la medida que la capacitación, perfeccionamiento y actualización refieran al conjunto de acciones dirigidas a los funcionarios de los que depende el desarrollo de la institución, entonces se comprenderá la importancia de la capacitación como herramienta para generar innovaciones y procesos de transformación. Por esto se sostiene que todo profesional debe poseer las siguientes características en su desempeño profesional: dominio de un saber legitimado, capacidad para diagnosticar problemas propios y encontrar por sí mismo soluciones; autonomía y responsabilidad individual con respecto a su tarea, y responsabilidad colectiva sobre el rendimiento de la institución.

Por otro lado, se establecen tres indicadores que permiten observar el desempeño del proceso fiscalizador en las entidades federativas, los cuales se refieren a la capacidad de las EFS en el desarrollo de las auditorías, a la cobertura de la fiscalización que llevan a cabo y a la efectividad de su proceso auditor. Estos indicadores y sus componentes contemplan principalmente las capacidades de las EFS y la forma en que se organizan y desarrollan las auditorías a los gobiernos estatales. Así, la distribución del poder político en los congresos locales y la calidad de las reglas formales explican las diferencias de las EFS en estos indicadores. (Gutiérrez Luna, 2015)
Estas características ponen de relieve lo impostergable de fomentar y desarrollar capacidades; bajo el significado que las capacidades son las habilidades, los conocimientos, las estructuras y las maneras de trabajar que hacen que una organización sea efectiva. Crear capacidades significa desarrollar cada una de las características mencionadas, con base en las fortalezas existentes y enfrentando los vacíos y deficiencias. Para ello, se requiere establecer un programa de capacitación coordinado, con el fin de incrementar la calidad profesional del personal auditor y, consecuentemente, mejorar los resultados de su labor. Se observa que estos esfuerzos están centrados en la capacitación, entendiendo las capacidades como el capital humano en el hacer de la fiscalización superior.

\section{Hacia una propuesta de profesionalización para las Entidades de Fiscalización Superior (EFS)}

Terence Nombembe, Presidente del Comité Directivo de la INTOSAI y Auditor General de Sudáfrica en 2014, declara:

"Estoy más convencido que nunca del papel tan valioso que juegan las Entidades Fiscalizadoras Superiores (EFS), no sólo en el fortalecimiento del gobierno público, sino también haciendo una diferencia significativa en la vida de los ciudadanos de nuestros respectivos países... La adopción de normas internacionales es sólo un elemento de lo que define a una Entidad Fiscalizadora Superior (EFS) como una institución verdaderamente profesional. Otro elemento clave es tener personal profesionalmente calificado...Es muy gratificante para una EFS ser la principal propietaria de su conocimiento y creación de capacidades.” (Nombembe, 2014:71)

En continuidad con los puntos anteriores, el diseño, desarrollo y evaluación de un programa de profesionalización, desarrollo y mejora continua de 
las Entidades de Fiscalización Superior, debieran partir de una detección de los vacíos, y deficiencias. Todo esto debe conseguirse a través de un diagnóstico integral de déficit, fortalezas y oportunidades de las capacidades institucionales. (Morales, 2014)

Sin embargo, esta tarea no es fácil, porque con frecuencia, la cultura de los organismos públicos no es proclive al aprendizaje y transformación permanentes; más aún si no se suman las voluntades ni los recursos indispensables para impulsar programas de capacitación y desarrollo profesional, aunque la finalidad de una gestión pública eficaz, íntegra, transparente, idónea debe asegurarse mediante propuestas de capacitación, desarrollo y mejora continua que aquilaten el cumplimiento de las responsabilidades por la producción de valor público, se reconozcan con mayor precisión los límites de su actuación y se responda por sus resultados. (Moreno, 2015)

La INTOSAI demanda que las EFS evalúen su valor y sus beneficios, tanto en términos de cómo llevan a cabo e informan sobre sus propias operaciones, como en términos de cómo agregan valor y mejoran la administración financiera pública en el entorno en que se desenvuelven. (Gómez Maldonado, 2012)

Además, con esta resolución los miembros de las Naciones Unidas toman nota con aprecio de la labor de la INTOSAI para fomentar la eficiencia, eficacia, rendición de cuentas y transparencia, así como la eficaz y eficiente recaudación y utilización de fondos públicos en el interés de los ciudadanos. (Organización de las Naciones Unidas, 2011)

\section{Conclusiones}

Las Entidades de Fiscalización Superior (EFS) son instancias que continuamente debe atender posibilidades de aumentar la profesionalización de su personal en la operacionalización de su visión y misión.

En este sentido, nos encontramos ante la oportunidad de diseñar, desarrollar y evaluar un programa integral, a modo de un ecosistema, que involucre diversos factores que formal o informalmente van configurando al auditor profesional.

La profesionalización del capital humano de las Entidades de Fiscalización Superior (EFS) es una necesidad advertida desde los últimos años, como lo declaran los Órganos Internacionales respecto a las Entidades de Fiscalización Superior mediante definiciones, declaraciones, juicios $\mathrm{y}$ hasta situaciones que exponen que deben generar mayor confianza; y que coadyuven en el desarrollo de los ciudadanos por la vigilancia constante del ejercicio de los recursos públicos.

En suma, las Entidades de Fiscalización Superior, se encuentran en una coyuntura de conocimiento, información, tecnología, reformas legislativas y orgánicas, que apuntan hacia la necesidad de que los auditores formen parte de los servidores o personal calificado, capacitado, con valores éticos, como un deber ser que influya positivamente en un ejercicio transparente y justo de los recursos públicos. 


\section{Referencias}

Instituto Mexicano para la Competitividad A.C. (IMCO) y el Centro Universitario de Ciencias Económico Administrativas de la Universidad de Guadalajara (CUCEA-UdG). (2013). Auditorías Superiores Locales en México: evaluación de su normatividad, prácticas y transparencia. México: IMCO-CUCEA.

Caruncho, L., \& Aiassa, M. (2016). Modernización del Estado y Capacitación: una mirada sobre sus alcances para la modificación de conductas y culturas Organizacionales. Revista de Investigación del Departamento de Humanidades y Ciencias Sociales Universidad Nacional de la Matanza, 1-25.

Cid, G. P. (2016). Comparativo institucional de las entidades de fiscalización en Centroamérica y el Caribe . Cotidiano, 71.

Figueroa Neri, A. (2007). La Auditoría Superior de México en el horizonte internacional de la fiscalización superior. Cd. de México: Auditoria Superior de la Federación.

Gómez Maldonado, M. A. (2012). ¿Qué competencias necesita el auditor para enfrentar los retos del presente? Auditoría y Gestión de los Fondos Públicos, 73-78.

Gutiérrez Luna, A. (2015). La fiscalización superior a nivel subnacional en México. Una evaluación del desempeño de las Entidades de Fiscalización Superior (EFS) locales . Revista Mexicana de Análisis Político y Administración Pública, 143.

Martínez Argüelles, S. (2017). La transformación de entidades de fiscalización superior: El caso del Colegio Internacional de Auditores de la OTAN. Auditoría y Gestión de los Fondos Públicos, 56.
Montilla Galvis, O. d., \& Herrera Marchena, L. G. (2006). El deber ser de la auditoria. Estudios Gerenciales, 83-110.

Morales, T. (2014). Los Retos para el Sistema Nacional de Fiscalización. Hitos de ciencias económico administrativas, 52-54.

Moreno, M. E. (2015). Entidades Fiscalizadoras Superiores y Accountability. Murcia, España: Universidad de Murcia .

Nombembe, T. (2014). El valor y beneficios de las EFS. Revista Técnica sobre Rendición de Cuentas y Fiscalización Superior, 71-83.

Organización de las Naciones Unidas. (2011). Resolución A/66/209. El sexagésimo sexto período de sesiones de la Asamblea General de las Naciones Unidas (págs. 1-2). New York, USA: ONU.

Organización internacional de las Entidades Fiscalizadoras Superiores (INTOSAI). (1977). Declaración de Lima. Declaración de Lima de Criterios sobre las Normas de Auditoría (pág. 9). Lima, Perú: INTOSAI.

Ortiz Ramírez, J. A., \& Cruz Pérez, J. A. (2017). Estudios / Workinn Papers. GIGAPP, 1-3.

Romero Gudiño, A. (2016). Los sistemas locales de fiscalización superior y sus desafíos. El mundo del abogado,

http://elmundodelabogado.com/revista/posiciones/item /los-sistemas-locales-de-fiscalizacion-superior-y-susdesafios.

Tiscareño Agoitia, R. N. (2016). El fortalecimiento de la fiscalización en México desde el poder legislativo. El Cotidiano, 53-64. 